\title{
Quality Control of Intensity Modulated Radiation Therapy (IMRT)
}

\author{
M.Rayhan Uddin ${ }^{1, *}$, Kushal Chanda ${ }^{1}$, M. Anwarul Islam ${ }^{2}$ \\ ${ }^{1}$ Department of Medical Physics and Biomedical Engineering, Gono University, Dhaka, Bangladesh \\ ${ }^{2}$ Square Oncology and Radiotherapy Centre, Square Hospital Ltd., Dhaka, Bangladesh
}

Copyright $(2017$ by authors, all rights reserved. Authors agree that this article remains permanently open access under the terms of the Creative Commons Attribution License 4.0 International License

\begin{abstract}
The aim of this study is to ensure the MLC positional and leaf speed accuracy. To check the MLC positional and leaf speed accuracy picket fence and synchronized segmented stripes test pattern were performed. The relative and absolute dosimetric verification were analyzed in this study. This project was followed by Quality control for Intensity-Modulated Radiation Therapy, as in the Recommendation No.15 from SGSMP. For relative dosimetric verification test such as different dose in same depth, same dose in different depth, chair test and inhomogeneous test were performed. All the plans were followed by Gamma index. To verify absolute dose 0.3 cc SemiFlex chamber along with a PTW solid water phantom was used. In picket fence and synchronized segmented stripes test, match-lines appear at -10.0, -5.0, 0.0, 5.0, 10.0 and $-12.0,-8.0,-4.0,0.0,4.0,8.0,12.0 \mathrm{~cm}$ respectively from the center of the field. The Gamma Index for the different dose in same depth, same dose in different depth, chair test and inhomogeneous test were $99.48 \%$ \& $-0.52 \%, 99.35 \%$ \& $-0.65 \%, 99.04 \% \&-1.96 \%$ and $98.34 \% \&-1.66 \%$ at the pixel range of -1.00 to $1.00 \& 1.00$ to 2.00 respectively. Calculated and measured absolute dose for three cases were 2.050 \&1.970 (\% deviation 4.06), 1.728 \& 1.730 (\% deviation -0.011) and $1.270 \& 1.250$ (\% deviation 1.6).
\end{abstract}

Keywords Intensity Modulated Radiation Therapy, Quality Assurance, Computerized Radiotherapy Treatment Planning System

\section{Introduction}

The process of changing the beam intensity profile to meet the goal of composite plan is called intensity modulated radiotherapy (IMRT). Multi leaf collimator (MLC) based intensity modulated radiotherapy is the result of a highly complex automated process of delivering dose to the patient. While linear accelerator and computer technology will inevitably proceed in the direction of self-checking and IMRT automation, quality control (QC) is still a vital component to ensure that the patient is treated accurately. IMRT refers to a radiation therapy technique in which non uniform fluence is delivered to the patient from any given position of the treatment beam to optimize the composite dose distribution [1]. Tumors and normal tissues are irradiated with modulated intensity beam in case of IMRT but in case of conventional radiotherapy tumors and normal tissue are irradiated with uniform radiation dose [2-6]. The positional accuracy of the MLC has a larger impact on delivered dose in IMRT than in conventional 3DCRT, where the MLC defines only the outer border of the beam. An uncertainty of 1-2 mm in leaf location may be clinically inconsequential in 3DCRT, but could have a large impact on the accuracy of IMRT delivery. Thus, the positional accuracy of the MLC should be evaluated over the full range of leaf travel and carriage motion that will be clinically employed. Quality assurance of the leaf speed, leaf position vs. time or monitor units, is only strictly necessary if performing IMRT with the dynamic MLC technique. With this technique however, exact control of leaf speed is the main condition determining the accuracy of IMRT delivery, and its measurement is therefore a critically important element of the IMRT quality assurance chain for dMLC delivery. Patient specific QA requires the possibility to apply the IMRT fields to a slab phantom and calculate the dose in that phantom. Depending on local resources and equipment, different QA checks may be performed, and it is the responsibility of the medical physicist to evaluate the needs [8, 10, and 12].

\section{Experimental Procedure}

\subsection{MLC Positional Accuracy and Leaf Speed}

According to Varian Medical System provided “ DMLC QA test patterns and procedures”, we have fixed the Gantry 
to $0^{\circ}$ Varian scale $\left(0^{\circ}\right.$ IEC scale) and the collimator to $0^{\circ}$ Varian scale ( $0^{\circ}$ IEC scale).Then retracted the MLC and a slab of plastic was placed on the treatment couch to provide a platform for the film. A verification film in a ready-pack envelope and a radio chromic film were used in this test. The film was large enough to record the tested field which has the size of 10 "/12". Film and solid water phantom were attached with white micropore tape. Then source to surface distance (SSD) was kept at $100 \mathrm{~cm}$ on film by vertically up the table and the crosshairs was marked above and below and to the right and left of the isocenter with small but detectable pinpricks. We also marked the upper right-hand corner of the film close to the gantry to identify the right placement of the film after development. Then 1A, 1B and1C each test were loaded into the MLC controller by using the stand-alone MLC workstation program for Picket Fence test [Test: 1] and synchronized segmented stripes [Test: 2]. In Test: 1 the match-lines between the $5 \mathrm{~cm}$ wide fields were straight and approximately equal in intensity and in Test: 2 the match-lines between the $4 \mathrm{~cm}$ wide fields were straight and approximately equal in intensity. Both tests were performed using the highest dose rate (500 MU/Min) that is normally used for clinical IMRT cases. A separate piece of film (Kodak X-OmatV for Picket Fence and GAFCHROMIC $\mathrm{RTQA}^{2} \mathrm{QA}+$ for Synchronized Segmented Stripes) was used for each test [8]. Stripes Picket Fence and Synchronized Segmented Stripes test pattern are given below:

Table 1. Picket Fence test

\begin{tabular}{|c|c|c|c|}
\hline File & Test $1 \mathrm{~A}$ & Test $1 \mathrm{~B}$ & Test $1 \mathrm{C}$ \\
\hline $\mathrm{X} 1$ & $20.0 \mathrm{~cm}$ & $5.8 \mathrm{~cm}$ & $-2.0 \mathrm{~cm}$ \\
\hline $\mathrm{X} 2$ & $-2.0 \mathrm{~cm}$ & $5.8 \mathrm{~cm}$ & $20.0 \mathrm{~cm}$ \\
\hline Y & $39.6 \mathrm{~cm}$ & $39.6 \mathrm{~cm}$ & $39.6 \mathrm{~cm}$ \\
\hline MU & 90 & 60 & 90 \\
\hline
\end{tabular}

Table 2. Synchronized Segmented Stripes test

\begin{tabular}{|c|c|c|c|c|}
\hline File & Test 2A & Test 2B & Test 2C & Test 2D \\
\hline $\mathrm{X} 1$ & $12.7 \mathrm{~cm}$ & $0.7 \mathrm{~cm}$ & $8.7 \mathrm{~cm}$ & $-0.2 \mathrm{~cm}$ \\
\hline $\mathrm{X} 2$ & $0.7 \mathrm{~cm}$ & $12.7 \mathrm{~cm}$ & $-0.2 \mathrm{~cm}$ & $8.7 \mathrm{~cm}$ \\
\hline $\mathrm{Y}$ & $24.4 \mathrm{~cm}$ & $24.4 \mathrm{~cm}$ & $24.4 \mathrm{~cm}$ & $24.4 \mathrm{~cm}$ \\
\hline $\mathrm{MU}$ & 80 & 80 & 40 & 40 \\
\hline
\end{tabular}

\subsection{Exactness of the Optimization of TPS}

The QA phantom with a holder was attached to CT couch and aligned with external laser and then moved the table 497 $\mathrm{cm}$ toward to align with CT scan gantry laser and the phantom was scanned at $0.5 \mathrm{~cm}$ spacing. All the scan images were transported to TPS server via DICOM media. We contoured as follows: Test: 3- Different doses at a same depth [Fig: 3], Test: 4-Same doses at a different depth [Fig: 4], Test: 5-chair test [Fig: 5], Test: 6- Inhomogeneous test [Fig: 6].In test: 3 and test: 4, we have drawn D1, D2 and D3 dose regions in the QA phantom both different doses at a same depth and same doses at a different depth. In test 1 , the amount of doses were D1 = 5000 cGy, D2 = 6000 cGy \& D3 $=7000 \mathrm{cGy}$ and in test 2 the dose was set to $5000 \mathrm{cGy}$ for each dose regions. In chair test, a shape like a chair in a QA phantom as shown in [fig: 5]. the amount of doses was $D=$ 7000cGy. For homogeneous test we contoured a solid materials into water in a phantom and drawn " $\mathrm{D}$ ” (Here, $\mathrm{D}=$ 7000cGy) which is fall half in water and half in solid and arranged 7 equal spacing fields and plan optimization was performed according to the desired dose regions by Eclipse 8.6 planning System. The dose rate was 500MU/MIN. Verification plan was created for every individual plan on scanned phantom along with IMRT matrix. Then the verification plans was delivered on IMRT matrix phantom. The calculated and measured dose of each verification plan was evaluated by gamma method.

\subsection{Absolute Dose Verification}

We have chosen a water-equivalent slab phantom (model: PTW-T29672/u 27-31016). The thickness of this slab phantom is $2 \mathrm{~cm}$. Another 18 slabs with $1.00 \mathrm{~cm}$ thickness each and one with $0.5 \mathrm{~cm}$ thickness were used. We selected "semi flex" ionization chamber. After setting up and scanning the phantom, the scan images were transferred to the scan images to TPS for creating verification plan. All the verification plans were delivered and doses were measured.

\section{Results}

\section{Test 1: Picket Fence}

The match-line includes a $1 \mathrm{~mm}$ gap. The match-lines appear at $-10.0 \mathrm{~cm},-5.0 \mathrm{~cm}, 0.0 \mathrm{~cm}, 5.0 \mathrm{~cm}$ and $10.0 \mathrm{~cm}$ from the center of the field. The match-lines fall within these limits.

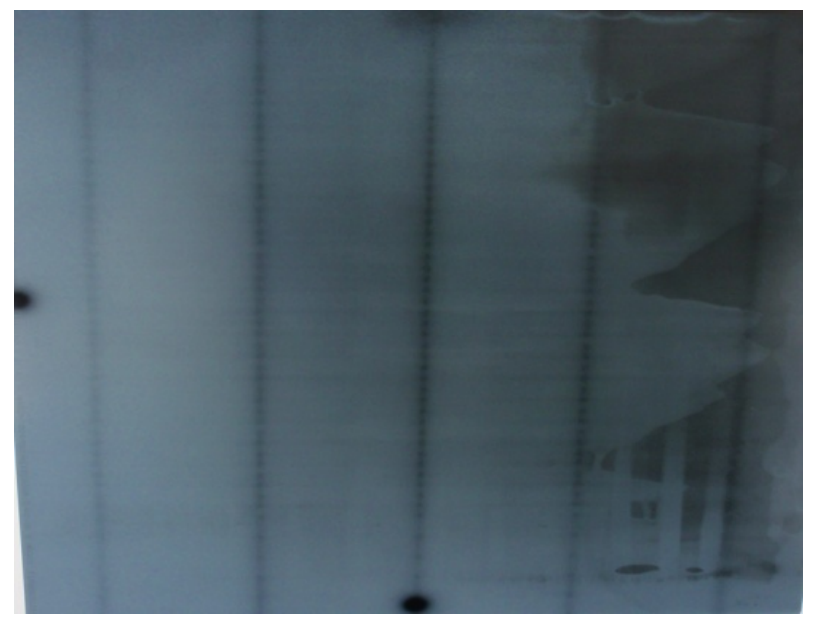

Test 1A, Test 1B, and Test 1C Delivered to one piece of film

Figure 1. Test1: Picket Fence 


\section{Test 2: Synchronized Segmented Stripes}

The match-lines appear at $-12.0 \mathrm{~cm},-8.0 \mathrm{~cm},-4.0 \mathrm{~cm}, 0.0 \mathrm{~cm}$, $4.0 \mathrm{~cm}, 8.0 \mathrm{~cm}$ and $12.0 \mathrm{~cm}$ from the center of the field. Intensity of all the exposed stripes is uniform.

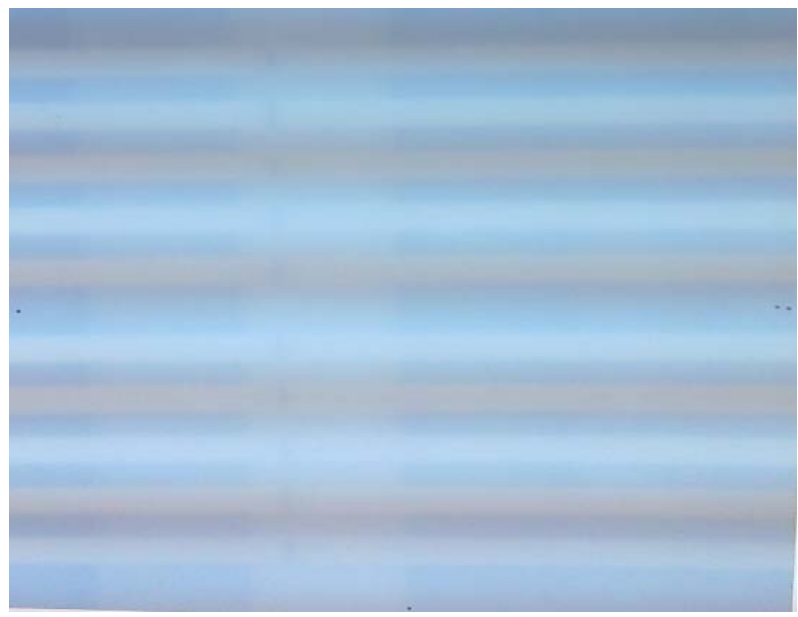

Test 2A, Test 2B, Test 2C, and Test 2D delivered to one piece of film

Figure 2. Test 2: Synchronized Segmented Stripes

\section{Test 3: Different Dose at Same Depth}

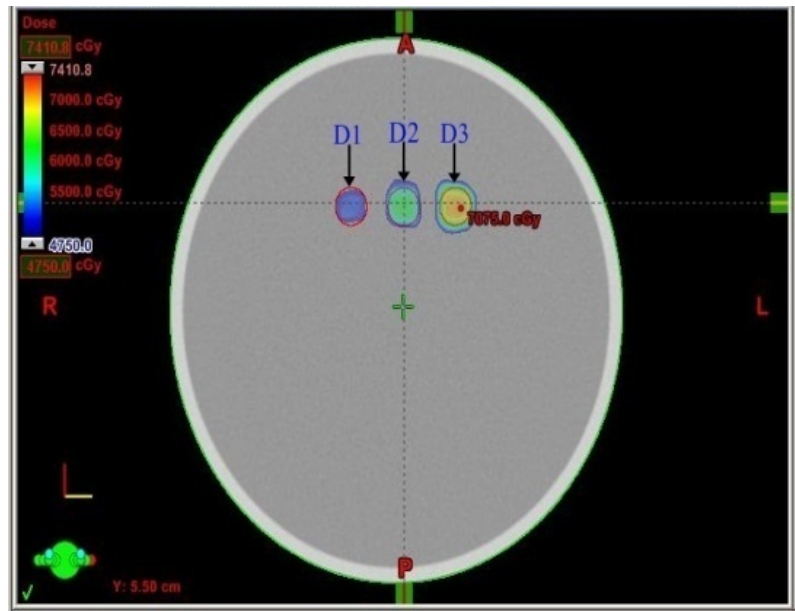

Figure 3. Different dose at same depth (plan)

Verification of Gamma Index for different dose in same depth (dose volume histogram)

Total number of pixels: 56169

Table 3. Different dose at same depth (percentage of pixels):

\begin{tabular}{|c|c|c|}
\hline Pixels in ranges & Number of pixels & Percentage[\%] \\
\hline-1.00 to 1.00 & 55878 & 99.48 \\
\hline 1.00 to 2.00 & 291 & -0.52 \\
\hline
\end{tabular}

\section{Test 4: Same Dose at Different Depth}

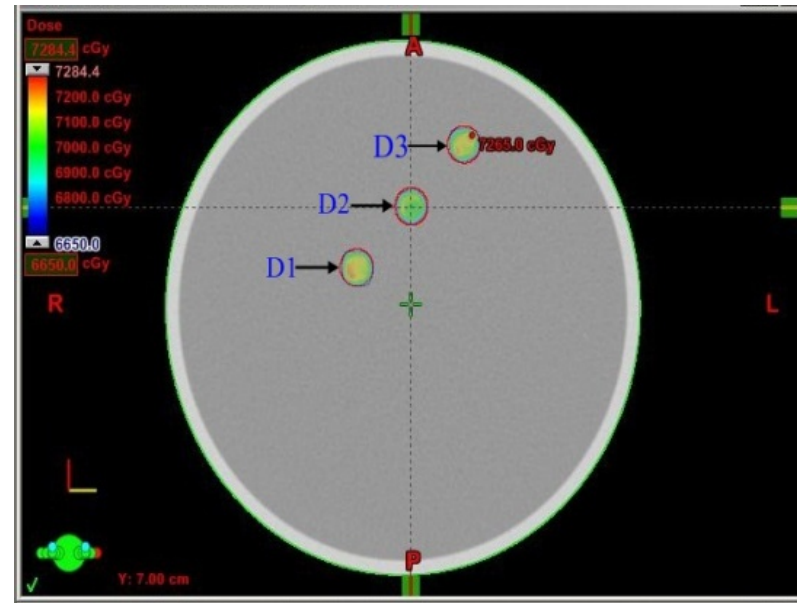

Figure 4. Same dose at different depth (plan)

Verification of Gamma Index for same dose in different depth (DVH)

Total number of pixels: 56169

Table 4. Same dose at different depth (percentage of pixels)

\begin{tabular}{|c|c|c|}
\hline Pixels in ranges & Number of pixels & Percentage[\%] \\
\hline-1.00 to 1.00 & 55806 & 99.35 \\
\hline 1.00 to 2.00 & 363 & -0.65 \\
\hline
\end{tabular}

\section{Test 5: Chair Test}

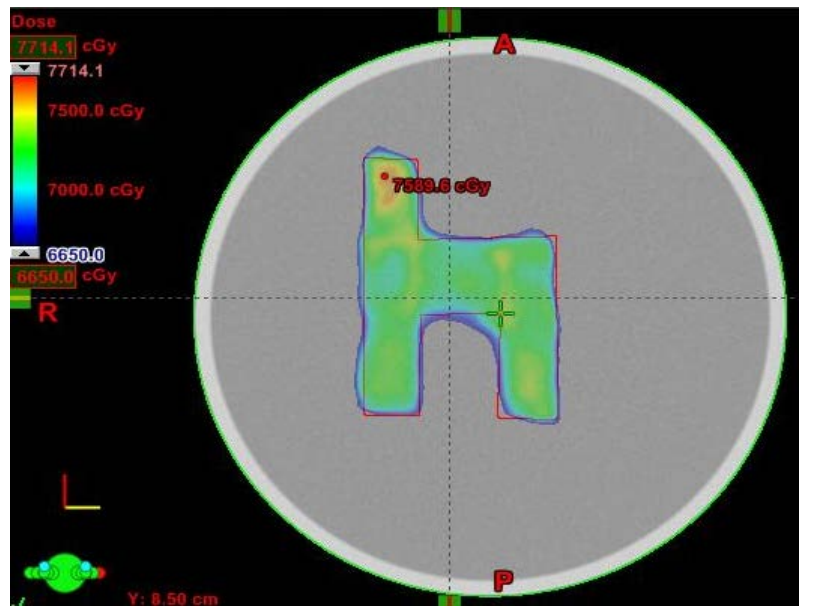

Figure 5. Chair test (plan)

Verification of Gamma Index for chair test (DVH) Total number of pixels: 56169

Table 5. Chair test (percentage of pixels):

\begin{tabular}{|c|c|c|}
\hline Pixels in ranges & Number of pixels & Percentage[\%] \\
\hline-1.00 to 1.00 & 55069 & 99.04 \\
\hline 1.00 to 2.00 & 1100 & -1.96 \\
\hline
\end{tabular}


Test 6: Inhomogeneous Test

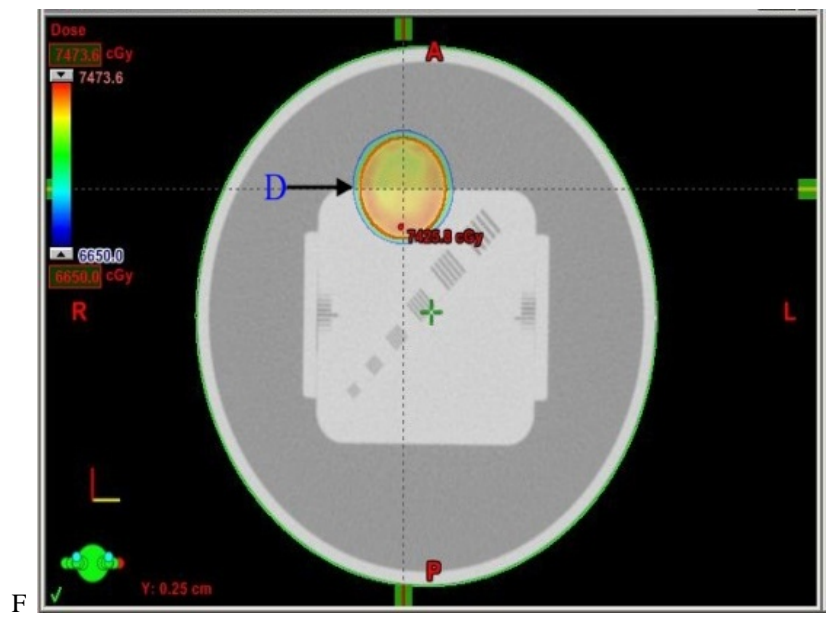

Figure 6. Inhomogeneous test (plan)

Verification of Gamma Index for Inhomogeneous test (DVH):-

Total number of pixels: 56169

Table 6. Inhomogeneous test (Percentage of pixels):

\begin{tabular}{|c|c|c|}
\hline Pixels in ranges & Number of pixels & Percentage[\%] \\
\hline-1.00 to 1.00 & 55238 & 98.34 \\
\hline 1.00 to 2.00 & 931 & -1.66 \\
\hline
\end{tabular}

\section{Test 7: The Planning of Absolute Dose Distribution Image}

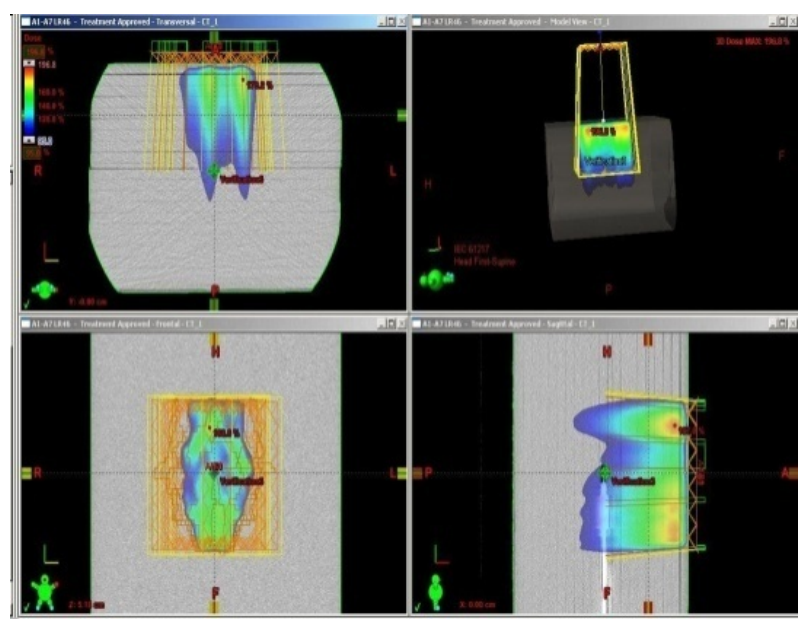

Figure 7. Treatment planning image after dose delivery on TPS

Table 7. Phantom and Chamber measurement:

\begin{tabular}{|c|c|c|c|c|}
\hline ID/ No. of Pt & $\begin{array}{c}\text { Delivered } \\
\text { dose (Gy) }\end{array}$ & $\begin{array}{c}\text { TPS } \\
\text { Calculation } \\
\text { (Gy) }\end{array}$ & $\begin{array}{c}\text { Practical } \\
\text { Calculation/ } \\
\text { measurement }\end{array}$ & Deviation \\
\hline R120604445 & 74 & 2.050 & 1.970 & 4.06 \\
\hline R120904010 & 56 & 1.728 & 1.730 & -0.011 \\
\hline R11022014 & 74 & 1.270 & 1.250 & 1.6 \\
\hline
\end{tabular}

\section{Discussion}

In the picket Fence test, normally the match-lines were appeared at -15.0 to $15.0 \mathrm{cmfrom}$ the center of the field. We used Kodak "X-omatV" film. It is smaller than other Kodak film. The match-lines appear at -10.0 to $10.0 \mathrm{~cm}$ from the center of the field, because of small size film. But, the result was accurate. On the other hand, the result of synchronized segmented stripes test was very accurate and the match-lines appear at -12.0 to $12.0 \mathrm{~cm}$ from the center of the field .We used micropore tape during the radiation delivery, to avoid unusual movement of film and solid phantom. In absolute dose measurement, we used semiflex ionization chamber because it has sensitive volumes and more suitability for use in water phantom. In different dose in same depth, same dose in different depth and chair test we have drawn the regions over QA phantom \& create verification planned and then doses were delivered.

\section{Conclusions}

Commissioning and quality assurance of dMLC for IMRT application requires considerable time and effort.The positional accuracy of the MLC has a larger impact on delivered dose in IMRT than in conventional 3DCRT, where the MLC defines only the outer border of the beam. Thus, the positional accuracy of the MLC should be evaluated over the full range of leaf travel and carriage motion that will be clinically employed. Leaf speed instability may arise from mechanical or steering problems or also due to the calibration technique employed. It is very important to ensure the MLC positional and leaf speed accuracy before the treatment is delivered in case of IMRT. The goal of this work is to ensure QC before implementing IMRT treatment. This report addresses different tests that should be performed during commissioning, acceptance and quality control of IMRT treatments. This report is an attempt to address some experiment setup and measurements that was followed by Recommendation No.15 from SGSMP. The accuracy and results of those test in this study showed that the quality control of IMRT were perfectly done.

\section{REFERENCES}

[1] https://www.slideshare.net/DilshadKP/intensity-modulated-ra diation-therapy-imrt

[2] Amn Van Esch; The paper Of "Experience from five radiotherapy departments" (according To acceptance test and QC procedures), Radiotherapy and Oncology 65; pp. 53-70; July 2002.

[3] A Zygogianni; Original research study of "Re-irradiation in head and neck cases using IMRT technique: A Retrospective Study with Toxicity and Survival Report”; pp. 1-5; Nov 2012. 
[4] Arun Chougule; PhD thesis "Study on Dosimetric Methods of Intensity Modulated Radiotherapy", Volume: 38, issue: 2; pp.109-110; May 2013.

[5] H A MCnair, E A Miles and C M Nutting; Review article of "Implementation of IMRT in the radiotherapy department". The British Journal of Radiology, 76 (2003), pp.850-856; Dec 2003.

[6] http://www.jmp.org.in/text.asp?2013/38/2/109/111337

[7] Intensity-Modulated Radiation Therapy for Breast and Lung Cancer: A Review of Use, Cost, Clinical Evidence, and Safety; CHRT Issue Brief; pp. 1-2; August 23, 2012.

[8] Swiss Society for Radiobiology and Medical Physics (SSRMP) Member of the European Federation of
Organisations for Medical Physics (EFOMP) and the International Organization for Medical Physics (IOMP); Quality Control of Intensity Modulated Radiation Therapy; Recommendations No. 15; September 2007.

[9] LoSasso T., Chui C.-S., Ling C. C., Physical and dosimetric aspects of a multileaf.

[10] Collimation system used in the dynamic mode for implementing intensity modulated radiotherapy, Med. Phys. 25, 1919-1927, 1998.

[11] www.elsevier.com/locate/radonline

[12] DMLC QA Test patterns and procedures; VARIAN medical system; P/N 10012878-01, Sep. 2001. 